
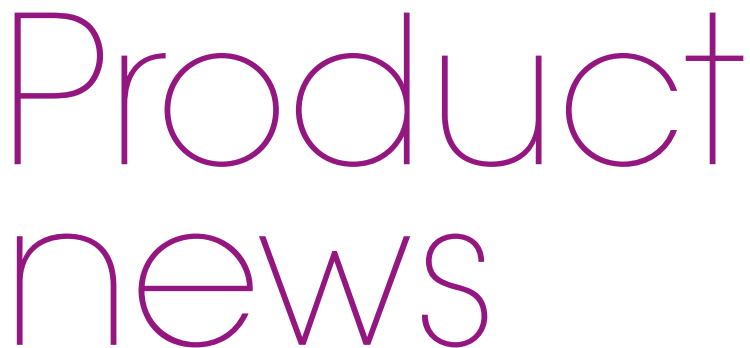

Product news is provided as a service to readers using text and images from the manufacturer, supplier or distributor and does not imply endorsement by BDJ Team. Normal and prudent research should be exercised before purchase or use of any product mentioned.

\section{A GAME-CHANGING NEW RANGE OF AUTOCLAVES}

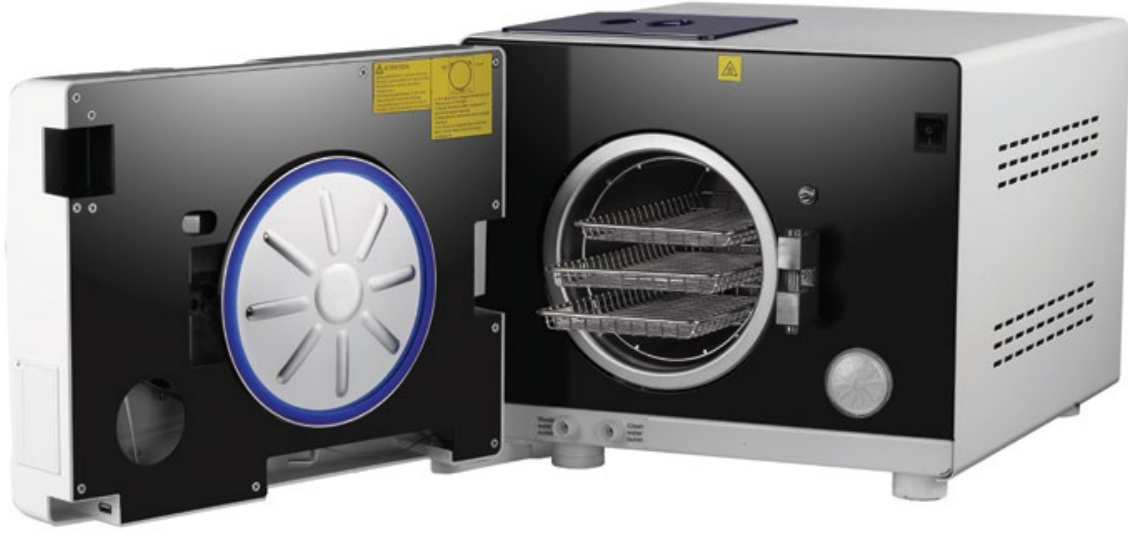

Yeson UK have launched a game-changing range of high pressure steam sterilisation autoclaves at prices as yet unseen in the dental practice sector. With dental practices under increasing pressure to acquire more patients and manage costs, the range is likely to generate a lot of interest.

The Yeson UK autoclave range performance matches that of the leading brands and delivers consistently reliable sterilisation at a much lower price point than the market has seen before, offering significant savings against other options. The entry level 8 litre machine retails at just $£ 1,100$.

All Yeson UK machines offer quiet Class B operation, 7 pre-set sterilisation programmes, B\&D Helix testing and a choice of printer or USB reporting. A range of sizes from 8 litre to 22 litre capacity offers an option for any size of practice. Compliance meets all required CE and BS standards.

For more information visit www.yeson-uk. com.
INCREASING EFFICIENCY AND SAFETY INTHE PRACTICE

High quality Galaxie Cassettes simplify the cleaning, sterilisation and classification of hand instruments. A stable case made of electropolished stainless steel protects the equipment, the unique design reduces the contact points. A laser-cut pattern maximises water through flow and increases efficiency in the RDG/thermal disinfector. Smooth rounded corners and slotted edges enhance drainage and shorten drying time. The ergonomic locking system can be operated with just one hand. The food-safe, colour-coded silicone rails can be assembled in any position and are compatible with all handle diameters. Available are DIN cassettes for 20 instruments, $1 / 2$ DIN cassettes for ten instruments and 1/4 DIN cassettes for five instruments. More information at www. am-eagle.de/en/galaxie-cassettes.

With the new Microbrush MB applicators dental fluids are dispensed precisely and economically. The flexible handles remain in the desired position without spring-back, the ultrafine 0.5 $\mathrm{mm}$ tip is ideally suited for pinpoint placement of substances. Non-absorbent fibres support the easy uptake and delivery of material. The useful tools are available in the colours orange and petrol, which enables the clear classification in multi-stage procedures. Samples can be requested at samples@microbrush.eu.

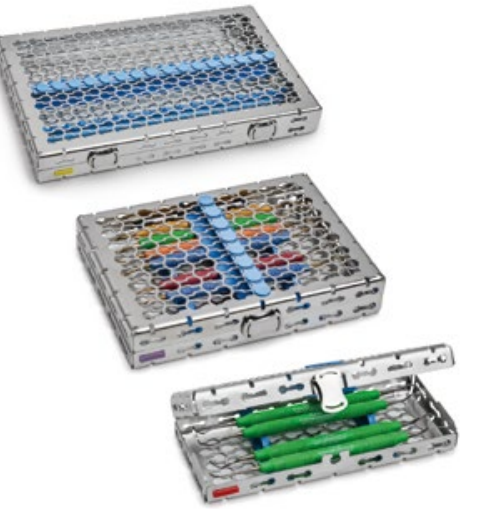

If you would like to promote your products or services direct to the dental industry in BDJ Team, call Andy May on 02078434785 or emaila.may@nature.com guidelines on how to treat periodontal disease. A lecture covering the updated IRMER regulations would be of interest to all members of the dental team, including dental nurses, as too would the lecture on the safeguarding of vulnerable adults. The BSDHT will have a stand at the event and are keen to engage with members. Dental nurses will be represented by the BADN as well as the SBDN.

To register visit dentalshowcase.com/

register. planning. She will include a treatment planning section, including the latest BSP 